\title{
Determination of Fracture Toughness for Small-Sized Specimens with Ultrafine Grain Structure
}

\author{
Ye. Ye. Deryugin ${ }^{1, \text { a) }}$, V. E. Panin ${ }^{1,2}$, and B. I. Suvorov ${ }^{1}$ \\ ${ }^{1}$ Institute of Strength Physics and Materials Science SB RAS, Tomsk, 634055, Russia \\ ${ }^{2}$ National Research Tomsk Polytechnic University, Tomsk, 634050, Russia \\ a) Corresponding author: dee@ispms.tsc.ru
}

\begin{abstract}
The results of changing the fracture toughness of materials are given for the VT6 titanium alloy. Its coarse grain structure was reduced down to ultrafine structure by forging. The specific energy of fracture $\gamma$ for the small-sized chevron-notched specimens was found thanks to the new technique. It is shown that severe plastic deformation reduces $\gamma$ at room temperature. The structure of the surface fracture of the VT6 alloy's ultrafine grain structure can be conceived as local zones of strong deformed material with a high concentration of pores. Such kind of structure cannot be formed only by means of crystallographic shears along close-packed planes. That fact proves the cardinal role of rotational modes of deformation in the origination and formation of a crack at various scale levels.
\end{abstract}

Keywords: ultrafine grain structure, localization of deformation, fracture, specific energy of fracture, titanium alloys

\section{INTRODUCTION}

Currently, there exists an actual problem of measuring the fracture toughness of materials for small-sized specimens where standard methods cannot be applied. The KI stress intensity factor (which characterizes inhomogeneous stress field in the vicinity of a crack tip in quasi-brittle materials) cannot be used as a parameter of crack resistance in metals and alloys where considerable plastic deformation precedes the origination and propagation of a crack. It means that the KI characteristic for a ductile material makes no physical sense, particularly for small-sized specimens.

A dependable characteristic of fracture toughness, irrespective of the nature of the material, is a critical value of rate of release of elastic energy $G_{\mathrm{c}}$ during crack propagation. This characteristic is equivalent to the J-integral [1, 2]. This energy, normalized on the surface area of the crack, is defined as specific fracture energy $\gamma$.

In that paper, fracture toughness was determined for the VT6 titanium alloys with coarse and ultrafine grain structures. A new technique developed in the laboratory of physical mesomechanics and nondestructive testing of ISPMS SB RAS was applied. The results of the research are analyzed on the base of a multi-level approach to physical mesomechanics.

\section{EXPERIMENTAL PROCEDURE}

A VT6 titanium alloy with ultrafine grain structure structure (the grain size of about $400 \mathrm{~nm}$ ) was used as the experimental material. The structure was formed via forging (strain is $50 \%$ ) at $400^{\circ} \mathrm{C}$ and tempering at $300^{\circ} \mathrm{C}$ over 1 hour. The specimens of a VT6 alloy with coarse grain structure (the grain size of $8-10 \mu \mathrm{m}$ ) were also tested.

The images of the lateral surface of specimens were captured once in two seconds by the PENTAX K-5 digital camera, which makes it possible to measure crack length and opening in the zone of a chevron notch. The fracture surface fractography was investigated via scanning electron microscopy.

International Conference on Physical Mesomechanics of Multilevel Systems 2014

AIP Conf. Proc. 1623, 111-114 (2014); doi: 10.1063/1.4898895

(C) 2014 AIP Publishing LLC 978-0-7354-1260-6/\$30.00 


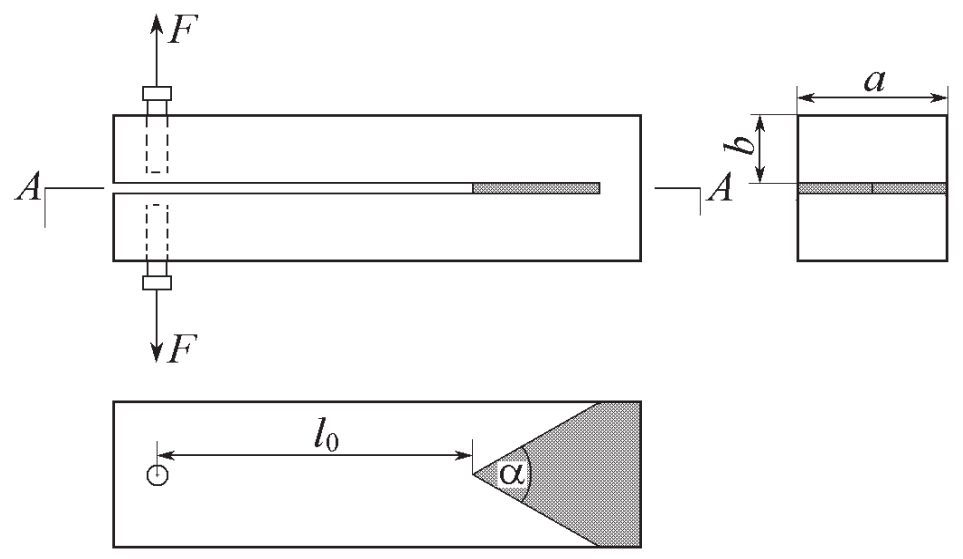

FIGURE 1. Scheme of the chevron-notched specimen

The samples were tested by applying the load at the rate of $v=2 \mu / \mathrm{c}$ at room temperature (Fig. 1). The specimens of $18 \mathrm{~mm}$ in length were cut from a square rod with the side of $6 \mathrm{~mm}$. The chevron notch was made using the electro-erosion technique as a narrow slot, with the width of $0.25 \mathrm{~mm}$. The notch boundary was designed as a Vshape line with angle $\alpha=\pi / 3$ (Fig. 1).

Specific fracture energy $\gamma$ was used as the main characteristic of fracture toughness, which was evaluated according to a new procedure for small-sized specimens with a chevron notch. The specimen was considered to be a small structure. The strain-stress analysis of the structure results in the following equation for the specific fracture energy:

$$
\gamma=\frac{12 F^{2} l^{2}}{E a^{2} b^{3}} f(\alpha, \Delta l),
$$

where $F$ is the applied external force. The function $f(\alpha, \Delta l)$ is defined by changing the contour of the notch in the process of crack development:

$$
f(\alpha, \Delta l)=\left[2+\frac{a}{l_{0}} \operatorname{ctg} \frac{\alpha}{2}\right]^{2} \frac{a k^{-1}}{4 \Delta l} \operatorname{ctg} \frac{\alpha}{2}\left(1-\frac{l}{3} k^{-1} \frac{d k}{d l}\right) .
$$

In relation (2) parameter $k$ equals to

$$
\begin{gathered}
k=\frac{2 \Delta l}{a} \operatorname{tg} \frac{\alpha}{2}\left[2+\frac{a}{l_{0}} \operatorname{ctg} \frac{\alpha}{2}\right]^{2}+\frac{l}{l_{0}}\left(1-\frac{2 \Delta l}{a} \operatorname{tg} \frac{\alpha}{2}\right)\left[4+\frac{a}{l_{0}} \operatorname{ctg} \frac{\alpha}{2}+\frac{2 \Delta l}{l_{0}}\right], \\
\frac{d k}{d l}=\frac{2}{a} \operatorname{tg} \frac{\alpha}{2}\left[2+\frac{a}{l_{0}} \operatorname{ctg} \frac{\alpha}{2}\right]^{2}+\frac{2 l}{l_{0}^{2}}\left(1-\frac{2 \Delta l}{a} \operatorname{tg} \frac{\alpha}{2}\right)+\frac{1}{l_{0}}\left[4+\frac{a}{l_{0}} \operatorname{ctg} \frac{\alpha}{2}+\frac{2 \Delta l}{l_{0}}\right]\left(1-\frac{2 l}{a} \operatorname{tg} \frac{\alpha}{2}(l+\Delta l)\right) .
\end{gathered}
$$

Here $\Delta l$ is the crack increment, $l=l_{0}+\Delta l$ is the current crack length.

One can see that, in contrast to standard formulas [3], these ones do not contain empirical constants and all necessary data can be obtained from the experiment.

\section{RESULTS}

Typical "force $P$ - displacement $\lambda$ " diagrams for a VT6 titanium alloy are shown in Fig. 2 for the coarse grain structure (a) and ultrafine structure (b) microstructures. In the alloy with coarse grains the crack originates at the tip of chevron notch at the very beginning of loading. Long before maximum applied force $F_{\max }$ is achieved, there is a long stage of stable crack growth, while the external force hardly changes. 


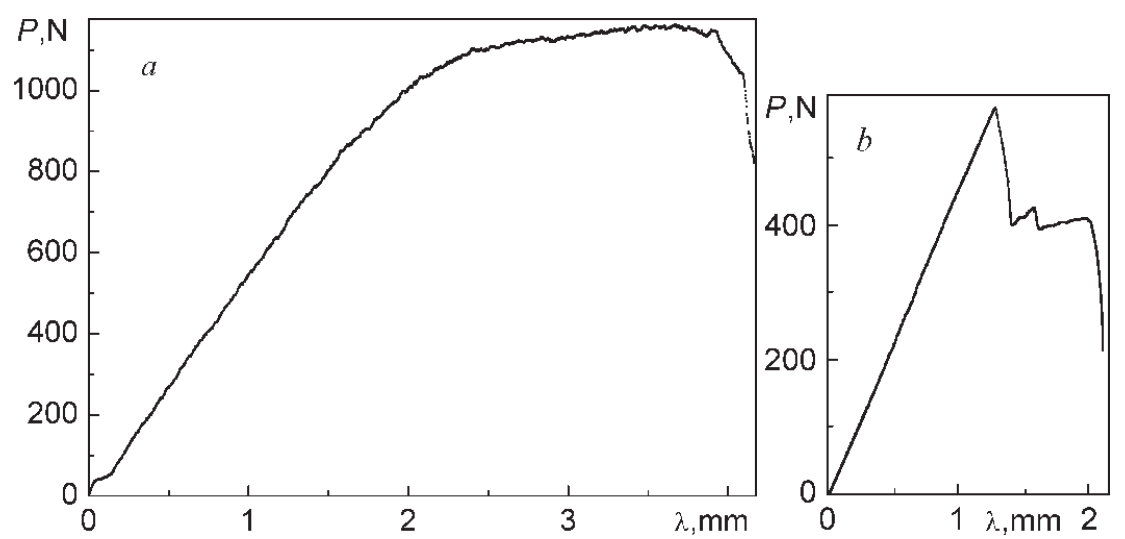

FIGURE 2. Loading diagrams for a VT6 alloy with the coarse grain (a) and ultrafine grain (b) structure

Another scenario was observed in case of a ultrafine grain material which is characterized by peak force in the diagram [4]. This peak shows the moment of crack origination at the tip of chevron notch. The "plateau" at $P-\lambda$ the curve corresponds to the stage of stable crack growth.

A comparison of the two diagrams proves that severe plastic deformation leads to decreasing crack resistance. Actually, calculations from Eq. 1 show that specific fracture energy for these two alloys is essentially different. Their critical values are 25.3 and $7.3 \pm 0.3 \mathrm{~kJ} / \mathrm{m}^{2}$, respectively.

Evaluations show that the measured value of opening $\lambda$ normally exceeds opening $\lambda^{\mathrm{e}}$ which is the result of releasing the elastic energy of crack growth. Additional variable $\lambda^{\mathrm{p}}$ is not related to crack growth and therefore does not refer to the compliance of the specimen. It is most appreciable for ductile materials.

In Fig. 3 the dependences of $\lambda^{\mathrm{e}}$ and $\lambda^{\mathrm{p}}$ versus $\lambda$ are shown for a VT6 alloy in the coarse grain state. It is seen that the contribution of $\lambda^{\mathrm{p}}$ is negligible at the initial stage of crack growth. The $\lambda^{\mathrm{p}}$ function grows practically linearly and its maximum value is close to the elastic deflection of the specimen $\left(\lambda^{\mathrm{p}} / \lambda^{\mathrm{e}} \approx 1\right)$. For the material with the ultrafine grain structure, the ratio is $\lambda^{\mathrm{p}} / \lambda^{\mathrm{e}} \leq 0.23$. Thus, the $\lambda^{\mathrm{p}}$ part of the plastic strain makes a significant contribution to the displacement, which is not associated with the formation of new surface cracks. It proves that there exists a large zone of plastic deformation in the front of the crack tip. It is known that the stress distribution here is significantly different as compared to the stresses of a Griffith crack in an elastic medium [5]. Therefore, the $K_{\text {Ic }}$ fracture toughness parameter loses its physical meaning.

The images of the fracture surfaces obtained via a scanning microscope provide additional information. In the surface image of the ultrafine grain VT6 alloy, one can see light bands with porous fiber structure (Fig. 4), which proves the severe plastic deformation in this region. The light band near the right boundary of the chevron notch (a) can be considered a plastically deformed material with an intricate porous structure. The fine structure of these bands in the ultrafine grain VT6 alloy reveals a great concentration of pores and a fragmentation of the material between the pores $(b, c)$. It indicates the essential role of the scale hierarchy of the rotational modes of deformation at the origination and formation of a crack in ultrafine grain materials. The crack originated at the chevron notch tip after a considerable local deformation which was accompanied by a local structure-phase degradation of the crystal structure [6]. The spatial distribution of these bands is the result of the

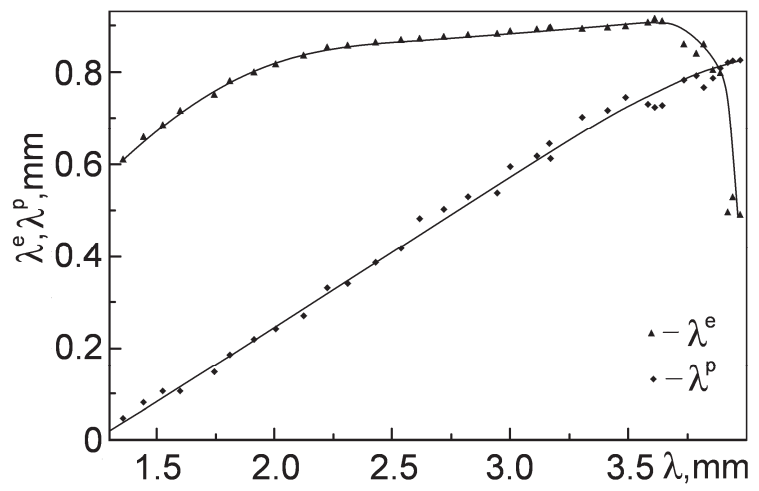
abrupt character of the crack growth.

FIGURE 3. The dependences $\lambda^{\mathrm{e}}$ and $\lambda^{\mathrm{p}}$ versus $\lambda$ 


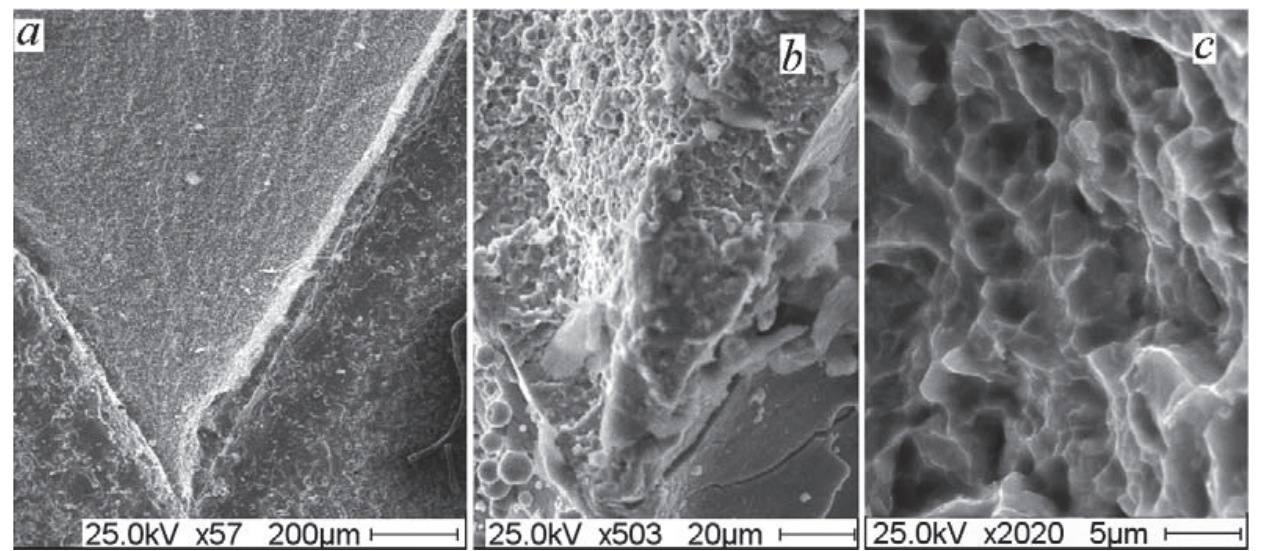

FIGURE 4. Structure of the localized deformation bands on the fracture surface of the ultrafine grain VT6 alloy

\section{CONCLUSION}

In this work specific fracture energies were found for a VT6 titanium alloy with coarse grain structure $\left(\gamma_{\mathrm{c}}=22 \pm 3 \mathrm{~kJ} / \mathrm{m}^{2}\right)$ and for one with the ultrafine grain structure $\left(\gamma_{\mathrm{c}}=6 \pm \mathrm{kJ} / \mathrm{m}^{2}\right)$ via testing small-sized specimens with chevron notches. The fractography of the fracture surface was also investigated by means of scanning electron microscopy. It is shown that the displacements of chevron notch tips have some additional part. This part is not related to a change in the compliance of the specimen at the crack growth stage (i.e. to the work of the applied external force that is supposed to form a new surface of the crack).

From the standpoint of physicals mesomechanics, the origination of the crack and its growth are the results of the two-phase degradation of a highly non-equilibrium material where one phase is a pore or a defect. Such kind of effects can emerge in the zones of local hydrostatic tension, which are characterized by an increased molar volume and whose thermodynamic Gibbs potential is very near to zero. From the very beginning, a material with the ultrafine grain structure is in a highly non-equilibrium state with an increased molar volume. On the other hand, boundary loading conditions produce a stress state with a high normal tension stress at the chevron notch tip which promotes a subsequent increase in the molar volume and the degradation of the material's structure. It is the cause of a considerable reduction in the fracture toughness of VT6 after severe plastic deformation.

The above mentioned band structure on the fracture surface in the regions of cracking retardation cannot arise from crystallographic shears along close-packed planes. It testifies in favor of the essential role of the rotational mode of deformation in the origination and formation of a crack in the hierarchy of various scale levels.

This work was supported by a research grant from the Russian Foundation for Basic Research (No. 13-0801404).

\section{REFERENCES}

1. R. W. Hertzberg, Deformation and Fracture Mechanics of Engineering Materials (Wiley, New York, 1989).

2. Yu. G. Matvienko, Models and Criteria of Fracture Mechanics (Fizmatlit, Moscow, 2006).

3. ASTM Designation E 399-09: Standard Test Method for Linear-Elastic Plane-Strain Fracture Toughness $K_{I C}$ of Metallic Materials (West Conshohocken, PA, USA, 2009).

4. Ye. Deryugin, Front. Eng. Mech. Res. 2(2), 28 (2013).

5. E. E. Deryugin and G. V. Lasko, J. Appl. Mech. Tech. Phys. 39(6), 934 (1998).

6. V. E. Panin and V. E. Egorushkin, Phys. Mesomech. 16(4), 267 (2013). 